\title{
An Entropy-Approach to the Duality Property
}

\author{
Salama Abdelhady \\ Department of Engineering, Canadian International College, Cairo, Egypt. \\ Email: salama_hady@cic-cairo.com
}

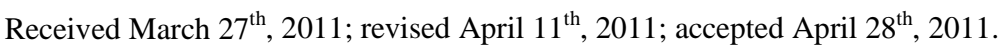

\begin{abstract}
According to a previously introduced entropy approach, it is possible to clarify the confusions of the duality concept that electrons and light may behave as waves or particles. In other words, the electron is clearly defined in this paper as a particle and the light is neatly defined as waves. Such an approach considered the flow of electric charges as a flow of ionized waves and the magnetic flux as electromagnetic waves of magnetic potential. By a similar entropy approach, the particle's kinetic energy is defined also as electromagnetic waves. So, the electron can be defined as an energized particle whose electric charge, magnetic energy and kinetic energy are forms of electromagnetic waves. According to these definitions and similarity of the mechanisms and laws characterizing the flow of mass and energy in general, the flow of electrons can be postulated as a simultaneous flow of two energy-components; particulized energy and wave energy. Hence, the electron doesn't have a dual nature. Rather, its behavior as a particle or as waves depends on the relative contributions of such components in the electron's flow. Reviewing the results of de-Broglie's experiments, it is possible to consider the flow of any particles as a simultaneous flow of waves and particles. Introducing the definition of the flow of electric charges as ionized waves, the photoelectric-effect can be postulated as an ionization process of the incident radiation during its reflection into an electric field. Similarly, the photovoltaic phenomena are postulated as a result of a photorefractive effect that may induce an electric potential into the incident radiation when crossing the electrically biased $p$-n junctions of photocells. Such postulates eliminate the confusing particle-property of light and prove that light has a wave-nature only. The truth of the introduced postulates is proven through finding plausible explanation of the sintering phenomena and thermoelectricity. Finally, this paper succeeded in introducing plausible explanations of results of Thompson's experiment and other phenomena that end the confusions in defining the true nature of light and electrons as waves and particles.
\end{abstract}

Keywords: Entropy, Duality Property, Electron, Light, Wave, Particle, Energy

\section{Introduction}

The propagation of waves through space is quite different from the propagation of particles. The flow of particles is governed by the laws of particle-mechanics while the flow of waves is characterized by Maxwell's equations of electromagnetism [1]. However, the propagation of particles was confused as a flow of waves to explain results of some experiments and the flow of light was also confused as a flow of particles to explain results of other experiments [2,3]. Such confusing explanations led to introducing the concept of "wave-particle duality" that stated the matter and light may exhibit the behaviors of both waves and particles depending upon the circumstances of each experiment [2]. Following an entropy approach [4], it is possible to clarify such confusions where the electron is clearly defined as a particle and the light is neatly defined as waves. Such approach defined the electric charge and magnetic flux as forms of energy or electromagnetic waves that possess electric or magnetic potentials [4,5]. Some references also define kinetic energy as a form of magnetic energy or electromagnetic waves $[6,7]$. By following the considered entropy approach, it was also possible to prove the nature of kinetic energy as electromagnetic waves. Accordingly, the electron can be defined as an energized particle whose electric charge, magnetic and kinetic energies are forms of electromagnetic waves. According to the similarity of the mechanisms and laws that govern mass and energy diffusions in addition to the Einstein's principle of mass energy equivalence $[1,8]$, it is possible to consider the flow of electrons as a simultaneous flow of two components belonging to two systems: a particulized energy system and a wave-energy system. The relative values of the wave energy and the particulized energy components define the electron's memberships to the two defined 
systems and describe the flow of electrons as a simultaneous flow of waves and particles [9]. Such definitions find plausible explanations of the duality property of electrons as discovered by the results of J. J. Thomson in the Cathode ray tube [10]. According to such a postulate; it is possible to introduce a plausible explanation of the solid state sintering-phenomena [11].

Introducing the flow of electric charges as a flow of ionized electromagnetic waves [4], the photoelectric effect can be postulated as ionizing the incident light waves during its reflection in the electric field of photoelectric tubes. Similarly, the photovoltaic phenomena are postulated as a result of a photorefractive effect that induces electric potentials into the incident radiation when crossing the electrically biased p-n junctions of photocells. Finally; it is possible to state that light has only one nature as electromagnetic waves and deletes the confusion of its dual nature as particles that bounce electrons.

Finally, this paper succeeded in introducing plausible explanations of results of Thompson's experiment and other phenomena that end the confusions in defining the true nature of light and electrons as waves and particles.

\section{Electric Charge and Magnetic Flux}

In a previous study, the time $\tau$ was replaced by the entropy $s$ in Maxwell's wave equations, and the electric charge was defined as a special solution of such Maxwell's equation. Such a solution is represented as follows [4]:

$$
\begin{gathered}
\boldsymbol{E}(\boldsymbol{r}, \boldsymbol{s})=\boldsymbol{s}(\varphi(\boldsymbol{r}, \boldsymbol{s}))+/-\Delta \bar{E} \\
\boldsymbol{E}(\boldsymbol{r}, \boldsymbol{s})=\boldsymbol{s}(\varphi(\boldsymbol{r}, \boldsymbol{s}))
\end{gathered}
$$

Equations (1) and (2) define the flow of electric charges as a flow of ionized electromagnetic waves which have a non-zero electric potential. The graphical representation of Equations (1) and (2) at specific position of the dimensional coordinate $\boldsymbol{r}$ is seen in Figures 1 and 2. Such figures show the electric potential $\boldsymbol{E}$ of such waves is oscillating, in the $\boldsymbol{E}$-s plane, around an initial non-zero value " $+/-\Delta \bar{E}$." Figure 1 represents a positive charge or an ionized electromagnetic wave that has a net positive potential. Similarly, Figure 2 represents a negative charge or an ionized electromagnetic wave of negative potential. The charge or energy in each imparted sinusoidal wave may have a net positive or negative potential according to the sign of the integral [5]:

$$
Q_{\text {elect }}=\int_{0}^{2 \pi} \bar{E} \mathrm{~d} S_{\text {elect }}
$$

Similarly; the following solution of the Maxwell's wave equations represent the magnetic flux as electromagnetic waves whose magnetic potential $\boldsymbol{H}$ is oscillating around a specified value of non-zero magnetic potential

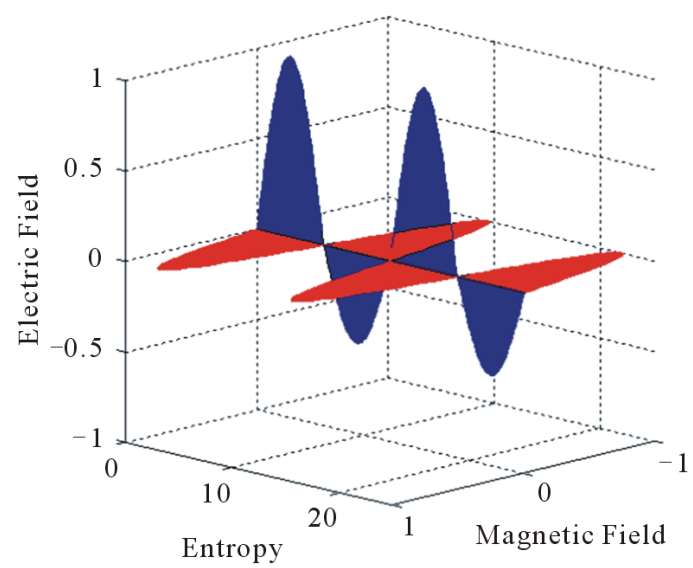

Figure 1. Graphical representation of a positive electric charge.

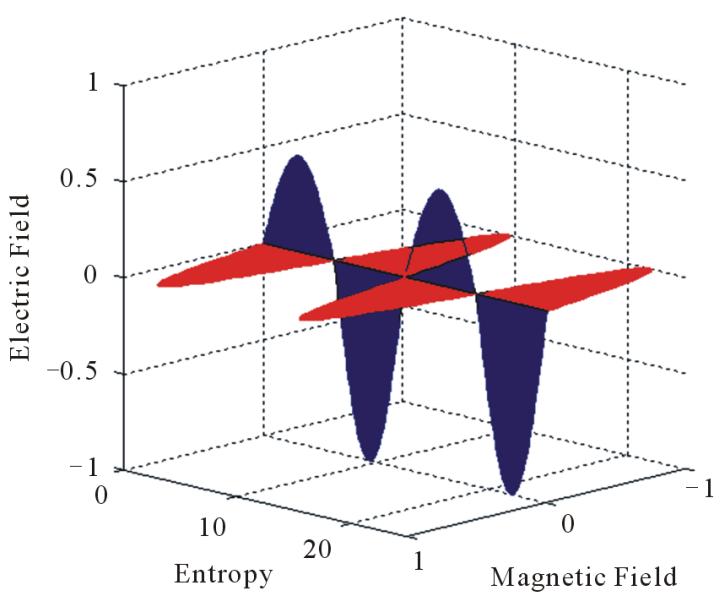

Figure 2. Graphical representation of a negative electric charge.

“ $+/-\Delta \bar{H}$ ” in the $\boldsymbol{H}$-s plane but its electric potential $\boldsymbol{E}$ is oscillating about a zero electric potential in the $\boldsymbol{E}$-s plane [4].

$$
\begin{gathered}
\boldsymbol{H}(\boldsymbol{r}, t)=\boldsymbol{s}(\varphi(\boldsymbol{r}, t))+/-\Delta \bar{H} \\
\boldsymbol{H}(\boldsymbol{r}, t)=\boldsymbol{s}(\varphi(\boldsymbol{r}, t))
\end{gathered}
$$

Equations (4) and (5) are represented graphically in Figure 3 where the net bounded area in the $\boldsymbol{H}$-s plane has a non-zero magnetic potential. So; the net value of the energy that has a magnetic potential or the magnetic flux has the flowing value [5]:

$$
\Phi=\int_{0}^{2 \pi} \bar{H} \mathrm{~d} S_{\text {mag }}
$$

Such postulates consider the electric charge and magnetic flux as energies that have specific potentials as the heat is considered as energy of specific thermal potential (temperature) [14]. 


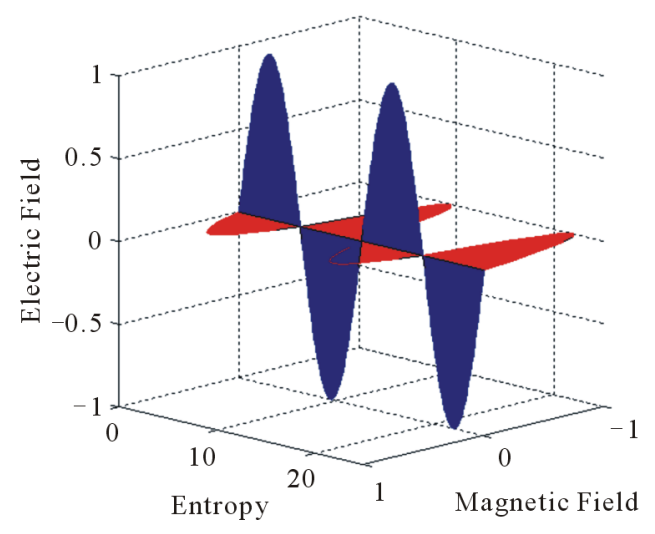

Figure 3. Graphical representation of magnetic flux.

\section{Kinetic Energy as a Form of Electromagnetic Waves}

According to the kinetic theory of ideal gases; the average molecular kinetic energy is proportional to the absolute temperature which is expressed by the following equation [15]:

$$
K . E .=1 / 2 m v^{2}=3 / 2 k T
$$

where $k$ is the Boltzmann's constant $=1.38 \cdot 10^{-23}$ joules $/ K$.

Equation (7) evaluates the kinetic energy of the molecules by its velocity or by its thermal energy which is defined by the temperature of the gas confining such molecules. The equation indicates common natures of kinetic energy that activates the motion of particles and thermal energy which flows as electromagnetic waves. Such similarity was also introduced by Toget [6] who found consistent equations that define the natures of kinetic energy and magnetic energy of the flowing electrons. Similarly; Rashkov proved also that kinetic energy is identical to electromagnetic energy and that Kinetic energy is conveyed from one object to another object in the form of electromagnetic waves [7]. So; it is possible to postulate that any flow of energy, in general, is measured by the same unit, Joule, and has one nature as electromagnetic waves.

\section{Similarity of the Flows of Mass and Energy}

The following equations estimate the force $\boldsymbol{F}$ between two mass particles $m_{1}$ and $m_{2}$ and two electric charges $Q_{1}$ and $Q_{2}$ separated by distance $\boldsymbol{r}$ [1]:

$$
\begin{gathered}
\boldsymbol{F}=\frac{m_{1} m_{2}}{4 \pi \boldsymbol{r}^{2}} \\
\boldsymbol{F}=\frac{Q_{1} Q_{2}}{4 \pi \boldsymbol{r}^{2}}
\end{gathered}
$$

Reviewing Equations (8) and (9); it can be observed that mass and energy are similar if we take into consideration that the electric charge, as postulated, is a form of energy.

Reviewing also the following laws that characterize the mass and energy diffusions as founded by Fick, Equation (10), and Fourier, Equation (11) [16]:

$$
\frac{\partial C_{A}}{\partial \tau}=D_{A B} \nabla^{2} C_{A}
$$

$D_{A B}$ : Molecular diffusivity; $C_{A}$ : Concentration of component $A$

$\tau$ : Time

$$
\frac{\partial \tau}{\partial \tau}=a \nabla^{2} T
$$

$a$ : Thermal diffusivity; $T$ : Temperature

The similarity of both Equations (10) and (12) indicates the equivalence of the mechanisms of transport of mass and heat energy. Equivalence of mass and energy is expressed also by the following relativity equation as stated by Einstein [8]:

$$
E=m c^{2}
$$

$m$ : Mass of the particle which is converted into energy; $c$ : Speed of light

So, the mass and energy are equivalent as expressed by Equation (12) and both have similar nature and transport mechanisms as shown in Equations (8-11).

\section{Simultaneous Flow of Particles and Waves}

The electrons are supposed to carry electric charge and magnetic moment and move around the nucleus of the atoms. According to the previous analysis, such forms of energy have a common nature as electromagnetic waves. So, an electron can be considered as an energized particle whose energy is in the form of electromagnetic waves.

Introducing the following definition of the relativistic kinetic energy K.E. [8]:

$$
\text { K.E. }=m_{e} c^{2}-m_{0} c^{2}
$$

where $m_{0}$ is the rest mass of an electron and $m_{e}$ is its relativistic mass. However; Equation (13) lacks other defined forms of energy that are associated by the flowing electrons, i.e. the electric energy E.E. and magnetic energy M.E. Such energies are in the form of electromagnetic waves as the kinetic energy. So, the term $E_{w}$ is introduced to define the total wave energy associated by the flowing electrons and it is expressed as follows:

$$
E_{w}=\text { K.E. E.E.+M.E. }
$$

Hence, Equation (13) should be modified as follows:

$$
E_{w}=\text { K.E. }+ \text { E.E. }+ \text { M.E. }=m_{e} c^{2}-m_{0} c^{2}
$$

So, the relativistic electron's mass can be defined as 
follows:

$$
m_{e}=m_{0}+\frac{E_{w}}{c^{2}}
$$

The rest mass of the electron is found in references as follows [17]:

$$
m_{0}=9.10953 \times 10^{-31} \mathrm{~kg} .
$$

This corresponds to the following amount of particulized or rest energy:

$$
m_{0}=m_{0} c^{2}=8.19 \times 10^{-14} \text { Joules }
$$

Assuming the electron is moving around the nucleus by half the velocity of light; its kinetic energy can be calculated as follows:

$$
\begin{aligned}
K . E . & =1 / 2 m_{0} v^{2}=1 / 29.10953 * 10^{-31} *\left(\frac{2.99 * 10^{8}}{2}\right)^{2} \\
& =1.016 * 10^{-14} \text { Joule. }
\end{aligned}
$$

According to the tabulated data of electron's properties [18]; the electron has also an electric energy of $4.1032 *$ $10^{-14}$ Joule and magnetic energy of $4.0842 * 10^{-14}$ Joule. Substituting such data into Equation (14), the total wave energy of an electron that flows by half the velocity of light will be as follows:

$$
\begin{aligned}
E_{w} & =(1.016+4.1032+4.0842) * 10^{-14} \\
& =9.2034 * 10^{-14} \text { Joule }
\end{aligned}
$$

Accordingly; the electron's energy has two components; particulized and wave energies. The sum of both components can be found as follows:

$$
\begin{aligned}
E_{e} & =E_{o}+E_{w}=(8.19+9.2034) * 10^{-14} \\
& =17.3934 * 10^{-14} \text { Joule }
\end{aligned}
$$

So, the ratios of the two components of the electron to the whole electron's energy, $E_{e}$, can be found as follows:

$$
\begin{gathered}
\mu_{o}=\frac{E_{o}}{E_{w}}=0.472 \\
\mu_{w}=\frac{E_{w}}{E_{e}}=0.528
\end{gathered}
$$

The values of the ratio of the two components $\mu_{o}$ and $\mu_{w}$ can be considered as memberships of the flowing electrons where $\mu_{o}$ is the particulized-system's membership and $\mu_{w}$ is the wave-system's membership. Such values indicate both the energy and mass ratios of the two components of an electron.

The considered electron has approximately equal values of particulized energy and wave energy. So; if the electron's velocity is half the velocity of light, half of the electron's mass will have wave's nature and the other half has a particle's nature. Accordingly; it is possible to postulate the flow of electrons may behave as particles or as waves according to its velocity or according to the relative values of its memberships. Such a postulate explains the dual behavior of the flow of electrons as a flow of particles or a flow of waves is not due to unknown nature of electrons but it is due to the simultaneously flowing waves and the electrons. The authenticity of such a postulate will be verified by reviewing the results of the Cathode-Ray experiment that were wrongly explained by some authors [10].

\section{Analysis of the Results of the Cathode-Ray Experiment}

During the latter half of the nineteenth century, it was found that while normally dry gases do not conduct an electric current, they do so under very low pressure and then patches of light are seen. Such passage of electricity through gases was studied by a number of physicists, particularly by Faraday, Davy, Crookes and J.J. Thomson [10]. When a current of high voltage (10,000 volts) is passed through a gas kept at a very low pressure $(0.01$ $0.03 \mathrm{~mm} \mathrm{Hg}$ ), blue rays are seen emerging from the case as seen by the dotted lines in Figure 4. These rays were called "Cathode Rays" and were defined later as electrons.

Such experiments had started by J. J. Thomson whose first experiment was to build a cathode ray tube with a metal cylinder on the end. This cylinder had two slits in it, leading to electrometers, which could measure small electric charges. He found that by applying a magnetic field across the tube, there was no activity recorded by the electrometers and so the charge had been bent away by the magnet. This proved that the negative charge and the ray were inseparable and intertwined. Then, he constructed a slightly different cathode ray tube, with a fluorescent coating at one end and a near perfect vacuum. Halfway down the tube were two electric plates, producing a positive anode and a negative cathode, which he hoped would deflect the rays. As he expected, the rays were deflected by the electric charge, proving beyond doubt that the rays were made up of charged particles carrying a negative charge. This result was a major discovery in itself, but Thomson resolved to understand more about the nature of these particles. Then, he decided to try to work out the nature of the particles. They were too small to have their mass or charge calculated directly, but he attempted to deduce this from how much the particles were bent by electrical currents, of varying strengths. Thomson found out that the charge to mass ratio was so large that the particles either carried a huge charge, or were a thousand times smaller than a hydrogen ion. He decided upon the latter and came up with the idea that the cathode rays were made of particles that emanated from within the atoms 


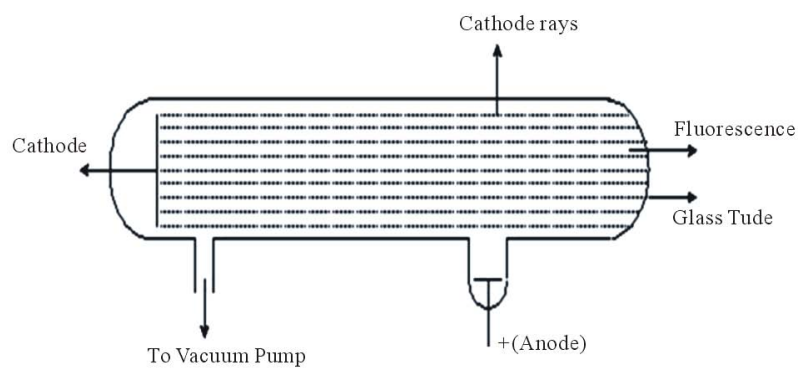

Figure 4. Cathode rays experiment.

themselves, a very bold and innovative idea. Thomson came up with the initial idea for the structure of the atom, postulating that it consisted of these negatively charged particles swimming in a sea of positive charge. However, the ideas of Thomson are still considered as facts that cannot be discussed. Such thinking led to open the way to the confusions of the nature of electrons as waves and particles.

Reviewing following properties of the cathode rays as found experimentally by J. J. Thomson and others [10]; it will be shown how the postulate introduced in this paper of considering flow of electrons as a simultaneous flow of particles and waves may offer more plausible explanations of the found experimental results than that the previous explanation of electrons as particles carrying ambiguous charges:.

- Cathode rays come out at right angles to the surface of the cathode and move in a straight line which is independent on the position of the anode. Such rays are energized electrons that leave the cathode surface in the direction of the anode. Their independent straightforward motion is a result of the directional momentum of the particulized component of the emerging electrons.

- They produce phosphorescence on certain salts like $\mathrm{ZnS}$ and fluorescence on glass and blacken photographic plates which result from the wave component of the flowing electrons.

- The rays pass through thin sheet of metals. If the metal sheet is too thick to be penetrated; the rays cast a shadow. Such characteristics may be produced by the electromagnetic-waves component of the flowing electrons.

- They produce X-ray when they strike a metal as may be done by the photorefractive effect on the electromagnetic-wave components of the flowing of electrons [18].

- The rays ionize a gas through which they pass as a result of the ionized component of the electrons-flow or the electromagnetic waves that have negative potentials.

- They heat a substance on which they fall according to the principles of energy conservation that absorbs the energy component of the flowing electrons or the kinetic energy of the particles.

- They rotate a light wheel placed in their paths. This shows that cathode rays contain material particles having enough momentum to rotate the wheel.

- The mass of a particle present in cathode rays is found to be $1 / 1837$ of $\mathrm{H}$-atom. This shows that the particle is of sub-atomic nature as done by particle component of the flow of electrons.

- Cathode rays are deflected by a magnetic or an electric field showing such flow as a simultaneous flow of particles and different forms of electromagnetic waves that possess electric and magnetic potentials.

- No cathode ray was produced when the tube was completely evacuated as this is a simultaneous flow of energy and mass diffusions. The absence of a medium of particles stops the diffusion of the particle component of the electron's flow

- Different gases produce the same cathode rays as they have the same e/m (energy/mass) ratio. This indicates that the particles present in cathode rays, i.e. electrons, are fundamental constituent of all matter.

So, these electrons and its associated energy represent a simultaneous flow of particles and electromagnetic waves. The ratio $\mathbf{e} / \mathbf{m}$ as found by J. J. Thompson, [10], can be considered as the membership of the wave or energy component in the flow of electrons, $\boldsymbol{\mu}_{w}$, and it may be used to find the kinetic energy or velocity of electrons inside the C.R.T.

\section{Analysis of the Photoelectric Effect}

Photoelectric effect is used to describe the process where light strikes a clean cathode plate. It is assumed, according to Einstein, that light behaves as particles or photons that eject the electron and cause the Ammeter deflection in the circuit shown in Figure 5 [1]. According to the previously postulated nature of electric charge as electromagnetic waves that have an electric potential, it is possible to find a more plausible explanation of such phenomena. When the incident light strikes the charged cathode plate inside the photo tube, Figure 5, the light will be ionized or gain a negative potential during its reflection by action of the tube-electric field.

However, the previously postulated nature of light as particles assumes that light is formed of photons that have energy of $h, v$ where $h$ is Planck's constant and $v$ is the frequency of light as a wave. If such photons will behave as particles whose momentum is the product of its mass and velocity, then the ratio of momentum of normal light photons, that have the wave length $0.55 \mu$, to the momentum of an electron moving at half speed of light, can be calculated as follows [8]: 


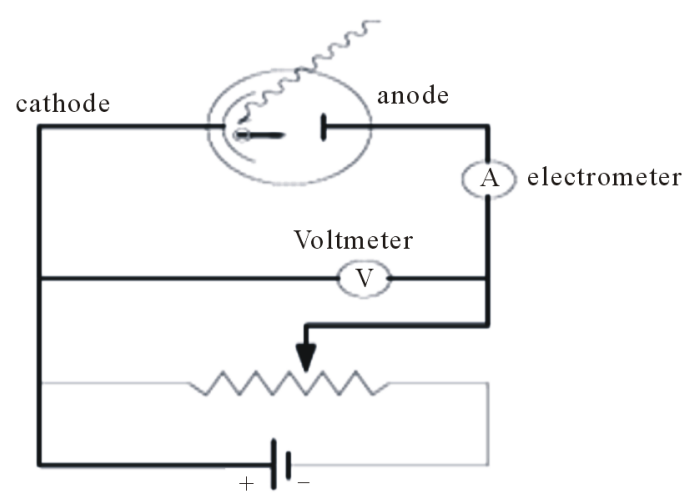

Figure 5. Photoelectric effect schematic.

$$
\begin{aligned}
\frac{(m * v)_{\text {photon }}}{(m * v)_{\text {electron }}} & =\frac{m_{\text {photon }}}{m_{\text {electron }}} * \frac{c}{c / 2}=\frac{2.25 * 1.602 * \frac{10^{-9}}{c^{2}}}{9.10953 * 10^{-31}} * 2 \\
& =0.0000009
\end{aligned}
$$

The result indicates to bounce one electron it requires more than 1 million of photons or light particles. However, it is more plausible to accept the postulate of waves may be ionized to form electric charges than to accept the possibility of bouncing an electron with more than a million of such postulated photons. Hence; the light is only a wave but it may gain an electric potential that ionizes it and converts it into a flow of electric charges.

\section{De Broglie Waves}

De Broglie speculated in 1924 that light is not the only matter which exhibits the confusing wave-particle duality [12]. He postulated that ordinary "particles" such as electrons, protons, or bowling balls could also exhibit wave characteristics in certain circumstances. According to de-Broglie, any particle of mass $\boldsymbol{m}$ which is moving at velocity $\boldsymbol{v}$ may emit electromagnetic waves of wavelength $\lambda$ that was found as follows [12]:

$$
\lambda=\frac{h}{\boldsymbol{m} * \boldsymbol{v}}
$$

$h$ : Planck's constant.

$\lambda$ : Wavelength

Relatively straightforward tests of Equation (19) were elaborated by diffraction and interference. A beam of such "particles" was shown at a diffraction grating and a diffraction pattern of a series of light and dark fringes results [12]. Hence, the wave picture of such postulate was adopted. For electrons, the sizes of slits required for the experiment were of the order of $10^{-11} \mathrm{~m}$ or so, which was readily available.

Such results may be considered as a proof of truth of the postulated nature of kinetic energy as electromagnetic waves and the flow of electrons or any particles as a simultaneous flow of waves and particles. However; the energy of such electromagnetic waves is equal, according to the postulated memberships, to the kinetic energy component of the flowing particles. Hence, such wave's energy can be determined from the kinetic energy according to the following Equation (20):

$$
\sum \frac{h * c}{\lambda}=1 / 2 m * v^{2}
$$

Reviewing de Broglie's Equation (19), which was verified experimentally, and Equation (20) which depends on the postulated nature of kinetic energy as waves, it can be seen that both equations are equivalent. According to Equations (19) and (20), the wave length of the electromagnetic waves emitted from moving particles is inversely proportional to the mass and velocity of such particles. So, de Broglie's hypothesis proves the generalization of the postulated flow of electrons as a simultaneous flow of particles and electromagnetic waves to any particle; i.e. to atoms or molecules. Accordingly, such a postulate may represent a starting point to analyze a previous study of Bose-Einstein condensation of atomic gases where atoms behave as waves [19].

\section{The Sintering Phenomena}

During sintering, the voids between crystals of some refractory materials, Figure 6, are filled when heat or electric current passes through the sintered material at temperatures below its melting point [9,11]. The mechanism of sintering was considered mainly due to mass diffusion. It is not plausible to assume mass diffusion through air-voids that separate the sintered grains at temperatures below the melting point of the sintered materials, Figure 6 [11]. The flow of particles is postulated as a simultaneous flow of particles and electromagnetic waves. So, it is possible to consider also the flow of heat or charges, during sintering, is a simultaneous flow of waves and particles that close the voids between the sintered particles. Accordingly; it is possible consider the flow of any waves or particles, in general, is a simultaneous flow of particles and waves.

\section{The Photovoltaic Effect}

The standard method to achieve conversion of incident solar radiation into electric charge is done through a p-type doped silicon wafer with a thin n-layer deposited on the surface [11], i.e. through p-n junction, Figure 7. The electronic mismatch between the $\mathrm{n}$ and $\mathrm{p}$-doped regions generates a potential bias across the junction and results in electrons and holes generated on their respective surfaces. The intermediate region is depleted of charge carriers-hence the term depletion region [18]. In 


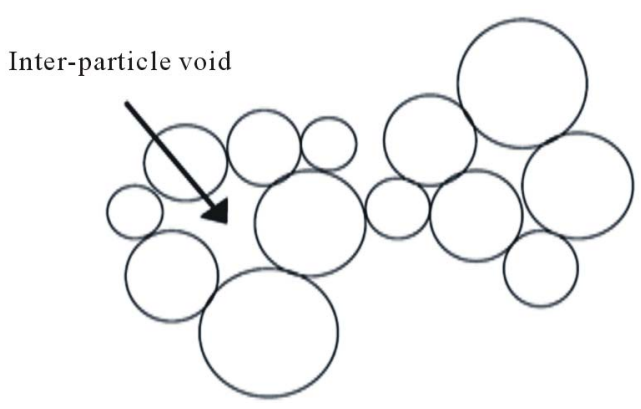

Figure 6. The voids between sintered particles.

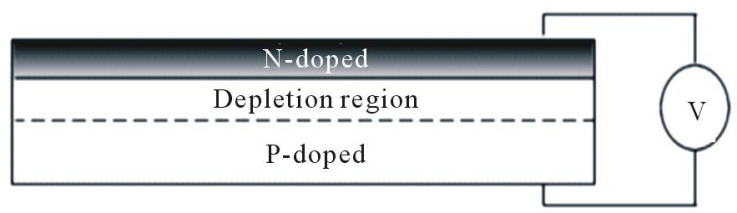

Figure 7. A standard photovoltaic device.

literature, the photovoltaic effect is done by bouncing the electrons in the n-doped regions by postulated light particles, photons, to fill the holes in the p-doped region [1].

However, the "photorefractive effect" was defined in previous literature to induce a change in the refractive index of materials through a combination of photovoltaic effect and electro-optic effect [20]. Accordingly, the photorefractive effect may induce an electric potential into the incident radiation when crossing the cell's p-n junction by the action of its potential bias. In other words, light gains the junction's potential when passing through photovoltaic cells by the photorefractive effect and behaves as a flow of electric charges.

Such postulate removes confusions regarding the duality of light waves that assume light has another nature as particles [8].

\section{Thermoelectricity}

Thermoelectricity was discovered and has been known since over a century ago [13]. In 1823, the German physicist Seebeck discovered that a voltage was developed in a loop containing two dissimilar metals, provided the two junctions were maintained at different temperatures, Figure 8. Scientific researches have not yet found a plausible explanation for the photovoltaic effect. However; the postulated nature of electric charge as electromagnetic waves of electric potential and the previously introduced photorefractive effect may help to explain the phenomena.

Due to the temperature difference between the tip and reference points, Figure 8, heat or electromagnetic waves flow through the thermocouple wires A and B. The difference of the Seebeck's coefficients of the two thermocouple wires induces an electrical potential into such

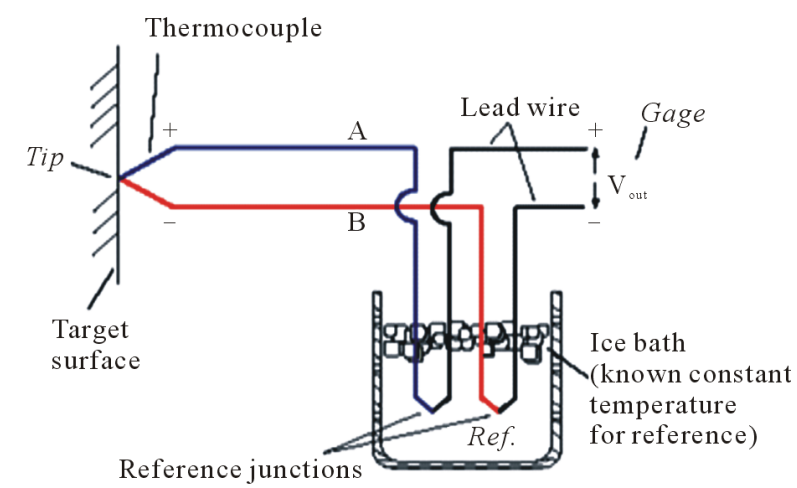

Figure 8. Thermocouple wiring.

waves when crossing the thermocouple junctions by a similar photorefractive effect [20]. Such induced potential is related to the thermal potential that drives the heat flow across the thermocouple junctions by the following Equation [21]:

$$
V_{\text {out }}=\left(S_{A}-S_{B}\right)\left(T_{\text {Tip }}-T_{\text {ref }}\right)
$$

So, the thermoelectricity assures the nature of a flow of electric charges as a flow of electromagnetic waves.

\section{Conclusions}

Following an entropy approach that defines the electric charge, magnetic flux and kinetic energy as forms of electromagnetic waves, it is possible to consider the flow of electrons or particles in general as a simultaneous flow of electromagnetic waves and particulized energy. According to this entropy approach, the photoelectric effect is considered also as ionizing reflection of the incident radiation in an electric field. Similarly, the photovoltaic phenomena are postulated as a result of the photorefractive effect that induces the potential bias across the photovoltaic junctions into the incident radiation. Such postulates clarify the confusion regarding the duality property of particles and light and lead to finding plausible explanations of other phenomena as sintering phenomena, de-Broglie hypothesis and thermoelectricity.

\section{REFERENCES}

[1] R. Haaiday, R. Resnick and J. Walker, "Fundamentals of Physics,” 7th Edition, John Wiley \& Sons, New York, 2004.

[2] D. Rothe, "Space and the Wave-Particle Engima," Ifinite Energy, the Magazine of New Energy Technology, Vol. 7, No. 42, 2002, pp. 49-57.

[3] L. Hackermüller, S. Uttenthaler and A. Zeilinger, et al., "The Wave Nature of Biomolecules and Fluorofullerenes,” Physical Review Letters, Vol. 91, No. 9, 2003.

[4] S. Abdelhady, "A Fundamental Equation of Thermodynamics that Embraces Electrical and Magnetic Poten- 
tials,” Journal of Electromagnetic Analysis \& Applications, Vol. 2, No. 3, 2010, pp. 162-166.

[5] S. Abdelhady, "Thermodynamic Analysis of Electric Charges and Magnetic Flux," Cairo 11th International Conference on Energy and Environment, Ghurgada, March 2009, pp. 175-185.

[6] C. V. Toget, "The Equivalence of Magnetic and Kinetic Energy,” Galilean Electrodynamics, Vol. 16, No. 6, 2006, p. 110.

[7] P. Rashkov, "Kinetic Energy is Identical to Electromagnetic Energy,” Philips-Universtat Marburg, 2010. http://processmodeling.org/theory/physics/kinetic.htm

[8] J. P. Wesley, “Classical Quantum Theory,” Weiherdammstrasse, Blumberg, 1996.

[9] S. Abdelhady, "Energy Analysis of the Electron Duality Property," Proceedings of the 14th International Conference on Aerospace Sciences \& Aviation Technology, ASAT-14, Cairo, May 2011, Paper MD-5.

[10] D. L. Anderson, "The Discovery of the Electron," Van Nostrand, Princeton, 1964.

[11] M. N. Rahaman, "Ceramic Processing and Sintering," 2nd Edition, Marcel Dekker, New York, 2003.

[12] P. A. Tipler and A. R. Leweyn, "Modern Physics," 4th Edition, W. H. Freeman and Co., New York, 2003.

[13] R. D. Rowe, “Thermoelectrics Handbook," Taylor \& Francis, London, 2006.
[14] A. C. Yunus and A. B. Michael, "Thermodynamics: An Engineering Approach,” McGraw-Hill Science Engineering, Boston, 2006.

[15] R. L. Liboff, “Kinetic Theory,” Prentice-Hall, Englewood Cliffs, 1990.

[16] D. M. William, "Analysis of Transport Phenomena," Oxford University Press, Oxford, 1998.

[17] W. M. Yao, “Review of Particle Physics," Journal of Physics G, Vol. 33, No. 1, 2006, pp. 77-115.

[18] J. S. Song and E. S. Yang, "A Study of the Photovoltaic Effect of a Semiconductor Grain Boundary by a Scanning Laser Beam,” Journal of Applied Physics, Vol. 58, No. 8, 1985, pp. 3129-3132. doi:10.1063/1.335816

[19] W. Ketterle, "When Atoms Behave as Waves; Bose-Einstein Condensation and the Atom Laser," Reviews of Modern Physics, Vol. 74, No. 10, 2002, pp. 1131-1151. doi:10.1103/RevModPhys.74.1131

[20] T. Sasaki, A. Katsuragi and Y. Nakazawa, "Photorefractive Effect of Power Stabilized Ferroelectric Liquid Crystals," International Conference on Optics and Photonics, India, November 2009.

[21] E. K. Iordanishvili, “Thermodynamic Potential of Thermoelectricity,” Journal of Thermoelectricity, Vol. 41, No. 1, 1999, pp. 5-12. 